\title{
Quero lhe usar! Uma Análise do Público-Alvo de Code Samples
}

\author{
Willian Braga ${ }^{1}$, Gabriel Menezes ${ }^{1}$, Awdren Fontão ${ }^{1}$, Andre Hora ${ }^{2}$, Bruno Cafeo ${ }^{1}$ \\ ${ }^{1}$ Faculdade de Computação - Universidade Federal de Mato Grosso do Sul (UFMS) \\ 79070-900 - Campo Grande - MS - Brasil \\ ${ }^{2}$ Departamento de Ciência da Computação - Universidade Federal de Minas Gerais (UFMG) \\ 31270-901 - Belo Horizonte - MG - Brasil

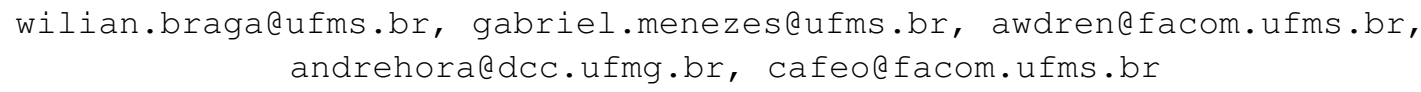

\begin{abstract}
Code samples are projects made available by organizations to assist the learning process of features of their products software. There is an increase in the importance that code samples have in the developer community, but little is known about who are using them. The target audience for code samples is believed to be the inexperienced developer, but to start a characterization, the current work presents an empirical study through the analysis of developer statistics on the Stack Overflow. As a result, implications have been generated that can assist organizations to keep code samples relevants to developers.
\end{abstract}

Resumo. Code samples são projetos disponibilizados por organizações para auxiliar no aprendizado de funcionalidades de seus produtos. Existe um crescimento na importância dos code samples para a comunidade de desenvolvedores, mas pouco se sabe sobre quem os usam. Acredita-se que o público-alvo dos code samples é o desenvolvedor inexperiente, mas para iniciar uma caracterização, este trabalho apresenta um estudo empírico por meio da análise de estatísticas de desenvolvedores no Stack Overflow. Como resultado, foi gerado um conjunto de implicações que pode auxiliar as organizações a manter os code samples relevantes para os desenvolvedores.

\section{Introdução}

A evolução de produtos/serviços de software, a competitividade e o atendimento à demanda de usuários direcionam organizações como Apple, Google e Spring a investir nas comunidades de desenvolvedores. Este cenário dinâmico, em um ambiente compartilhado em torno de uma plataforma central, tem sido estudado como Ecossistema de Software (ECOS) [Bosch 2009]. Para apoiar as contribuições dos desenvolvedores em ECOS, essas organizações disponibilizam os chamados code samples. Code samples são projetos de software armazenados em repositórios de código para apoiar a aprendizagem, por exemplo, de: funcionalidades de frameworks, bibliotecas e APIs [Menezes et al. 2019]. O Android, por exemplo, tem mais de cem code samples no GitHub para ajudar os desenvolvedores a criar aplicações para dispositivos móveis ${ }^{1}$. O mesmo acontece com o $\mathrm{AWS}^{2}$

\footnotetext{
${ }^{1}$ https://github.com/googlesamples

${ }^{2}$ https://github.com/aws-samples
} 
e Azure ${ }^{3}$, que disponibilizam code samples para auxiliar os desenvolvedores a lidar com serviços de computação em nuvem.

Apesar da crescente importância, existem poucos trabalhos na literatura técnica sobre code samples. Por exemplo, há estudos que exploram as características mais comuns de code samples e projetos similares [Menezes et al. 2019, Zhang et al. 2019]. Nesses trabalhos, destaca-se, por exemplo, a simplicidade e baixa complexidade do código disponibilizado. Apesar de se conhecer algumas características importantes e recorrentes de code samples, ainda sabe-se pouco sobre o perfil dos desenvolvedores que os buscam e os utilizam. A informação sobre que tipo de desenvolvedor utiliza code sample pode ser útil, pois: (1) a organização busca a disseminação do conhecimento para comunidade e a aceleração de contribuições (incluindo evolução) em torno de seus produtos; e (2) a comunidade de desenvolvedores busca contribuir na "curadoria" do conteúdo, visto que, compreenderão os perfis de desenvolvedores que utilizam code samples. Nesse contexto, é importante caracterizar os desenvolvedores que fazem uso desses code samples. Caso os desenvolvedores que utilizam os code samples sejam pouco experientes, as organizações devem se preocupar em criar code samples de baixa complexidade e com funcionalidades mais simples. Por outro lado, caso os desenvolvedores possuam maior experiência, os code samples podem apresentar, por exemplo, funcionalidades mais complexas e que possibilitem a evolução das contribuições do desenvolvedor. Todavia, acredita-se que o público-alvo dos code samples são desenvolvedores inexperientes em programação.

Como forma de avançar na direção de uma caracterização dos desenvolvedores que utilizam code samples, esse estudo inicial explora o grau de experiência de uma amostragem desses desenvolvedores por meio da reputação em um site de Q\&A ${ }^{4}$ (Stack Overflow). A reputação é item relevante, por exemplo, no GitHub para aceitação de pull requests [Amreen et al. 2019] e no Stack Overflow (SO) que libera desenvolvedor de restrições na criação de uma publicação [Fontão et al. 2017]. Nesse estudo foram analisadas as reputações de desenvolvedores envolvidos em 1.188 perguntas e respostas (relacionadas a 607 code samples do Google Android, Spring Boot, Amazon AWS e Microsoft Azure) do SO. Os principais resultados obtidos foram: (i) os code samples são relevantes para desenvolvedores experientes e inexperientes; (ii) os desenvolvedores que utilizam code samples não são tão inexperientes como poderia se pensar; na verdade, são mais experientes que a média da comunidade do Stack Overflow; (iii) para poder sanar as dúvidas relacionadas aos code samples, é necessário que, a comunidade de desenvolvedores que utilizam code samples possua indivíduos mais experientes.

\section{O que são code samples?}

A Oracle diz que "code sample são fornecidos com propósitos educacionais e para auxiliar o seu desenvolvimento ou controle de esforços". . Já o Spring relata que "code samples

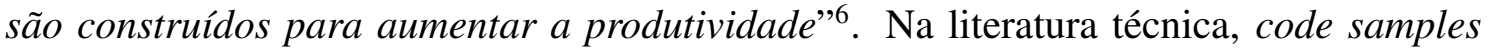
são definidos como projetos de software completos disponibilizados por organizações em repositórios de código oficiais com o propósito de auxiliar desenvolvedores no entendimento de um determinado conceito de um produto ou tecnologia [Menezes et al. 2019].

\footnotetext{
${ }^{3}$ https: / /github.com/Azure-Samples

${ }^{4}$ Plataforma de perguntas e respostas

5 https://www.oracle.com/technetwork/indexes/samplecode

${ }^{6}$ https://spring.io/guides
} 
Devido ao carácter educacional e de produtividade, code samples devem simples, pequenos e fáceis de serem utilizados [Menezes et al. 2019]. Observa-se que isso acontece com os code samples analisados neste trabalho. A Figura 1 apresenta métricas relacionadas ao tamanho e à complexidade dos code samples estudados. Percebe-se que, de maneira geral, os code samples são pequenos e simples em termos de número de arquivos, linhas de código por arquivo e complexidade ciclomática.

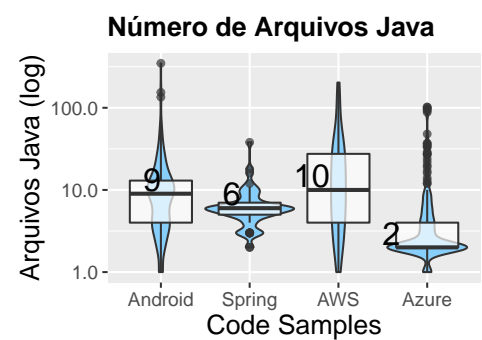

(a)

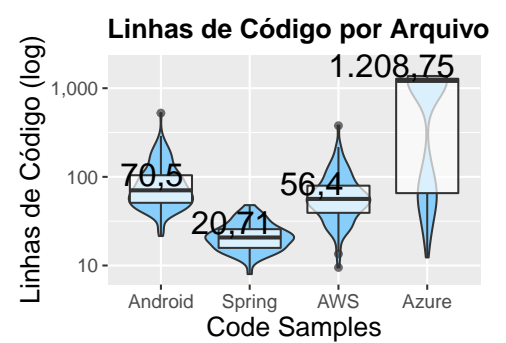

(b)

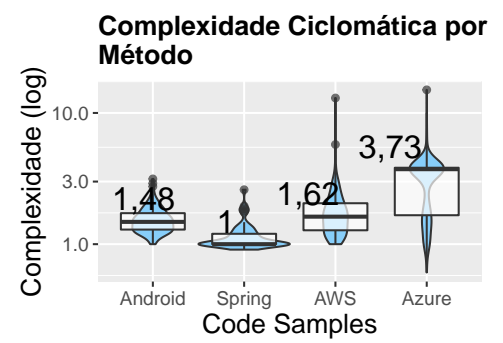

(c)

Figura 1. Métricas dos code samples

Como exemplo, a Figura 2 apresenta parte de um code sample oficial desenvolvido pelo Spring Boot ${ }^{7}$. De maneira geral, ele visa auxiliar os desenvolvedores no entendimento da construção de uma aplicação web utilizando o Spring Boot. Esse code sample possui 37 arquivos, sendo 6 relativos a código-fonte (com um total de 112 LOC) e 29 de outros arquivos (como arquivos jar, build.gradle, pom.xml e travis.yml).
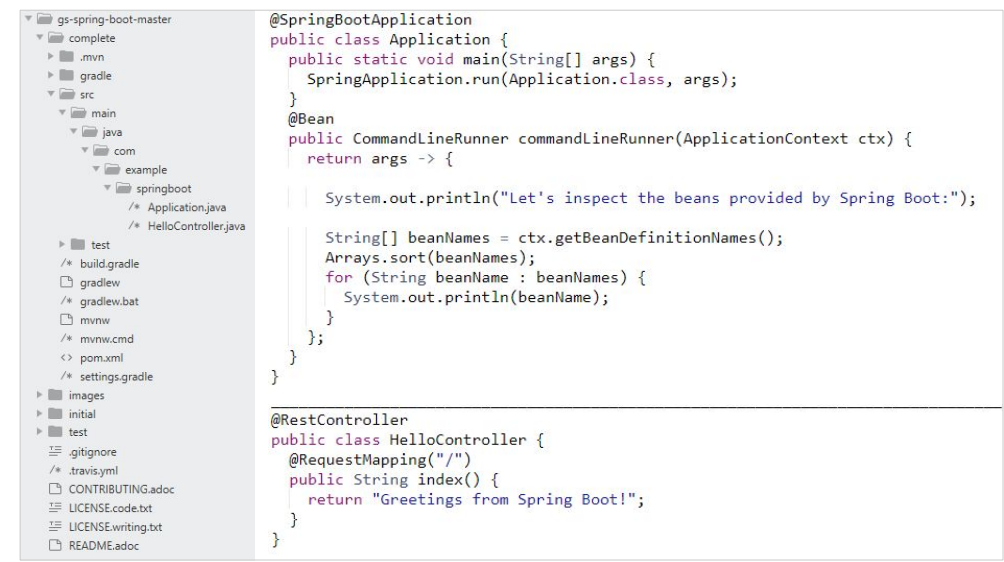

Figura 2. Estrutura de diretórios e trechos do código de um code sample do Spring Boot.

Esse code sample também possui 533 estrelas e 1.724 forks $^{8}$ no GitHub ${ }^{9}$. Devido ao seu tamanho reduzido e simplicidade acredita-se que ele seja relevante e útil, sobretudo aos desenvolvedores iniciantes. Além disso, destaca-se que este é um projeto ativo e que evolui ao longo do tempo (199 commits e 21 releases) e possui atividade na comunidade de desenvolvedores (5 perguntas no SO que possuem 6 respostas).

\footnotetext{
${ }^{7}$ https://github.com/spring-guides/gs-spring-boot

${ }^{8} \mathrm{Um}$ fork é uma cópia do repositório que permite que sejam realizadas alterações sem afetar o projeto original.

${ }^{9}$ https://github.com/spring-guides/gs-spring-boot
} 


\section{Estudo Exploratório}

Esta seção tem como objetivo apresentar os procedimentos realizados ao longo do estudo. A Seção 3.1 apresenta os objetivos do estudo. Já a Seção 3.2 mostra como foi a seleção dos code samples. Por fim, a Seção 3.3 apresenta como foi realizada a coleta das métricas.

\subsection{Objetivo do estudo e questões de pesquisa}

Este trabalho busca explorar a reputação dos desenvolvedores que perguntam sobre o code sample, bem como a dos que respondem sobre o code sample. A reputação pode, também, estar relacionada com a experiência e perícia do usuário [Bosu et al. 2013, Morrison and Murphy-Hill 2013]. A ideia é utilizar a reputação como um dos indicadores de experiência e conhecimento dos desenvolvedores. Com isso, pretende-se ter um intervalo que pode indicar o nível de experiência dos desenvolvedores que usam code samples. Ou seja, consegue-se saber qual a reputação esperada dos desenvolvedores que têm dúvida sobre o code sample, bem como a de desenvolvedores que conseguiram entender as dúvidas sobre os code samples (i.e., quem respondeu às perguntas).

Dessa forma, organizações podem definir melhor o público-alvo dos seus code samples e adaptar características dos code samples para melhor atendê-los. Para isso, neste trabalho, investiga-se as seguintes questões de pesquisa: [Q1] Qual a reputação dos desenvolvedores que perguntam sobre code samples no $\mathrm{SO}$ ?, e [Q2] Qual a reputação dos desenvolvedores que respondem as perguntas sobre os code samples no SO?

\subsection{Seleção de Code Samples}

Foram selecionados produtos de quatro organizações: Google, Spring, Amazon e Microsoft. Mais especificamente, foram selecionados 176 code samples do Android, 57 de Spring Boot, 111 do AWS e 263 do Azure, totalizando 607 code samples. Os motivos que levaram a esses code samples são: (1) vasta gama de desenvolvedores que os utilizam; (2) ajudam na criação de variados nichos de aplicações: dispositivos móveis, aplicações web e computação em nuvem; (3) estão disponíveis no GitHub; (4) code samples na linguagem Java que são os mais numerosos nos repositórios das organizações escolhidas. A seleção dos code samples ocorreu por meio da busca fornecida pela interface gráfica do GitHub $^{10}$ na página de repositórios aplicando o filtro para projetos em linguagem Java.

\subsection{Procedimento de Coleta de Perguntas, Respostas e Reputação}

Procedimento de coleta. Para identificar perguntas do SO que são relacionadas aos code samples, se utilizou a seguinte abordagem: uma pergunta é relacionada a um code sample quando é encontrado no seu corpo uma referência à URL do seu repositório no GitHub. Uma resposta está relacionada quando ela for resposta de uma pergunta relacionada ao code sample. Todo o procedimento de coleta das perguntas e respostas foi por meio da API oficial do Stack Exchange ${ }^{11}$ e da biblioteca StackAPI ${ }^{12}$. O período de coleta de perguntas e respostas compreende o período: Novembro de 2013 até Junho de 2020. Os dados utilizados na pesquisa está disponível para download ${ }^{13}$.

\footnotetext{
${ }^{10}$ Exemplo de busca: https://github.com/azure-samples? language=java

${ }^{11}$ https://api.stackexchange.com/

${ }^{12}$ https://stackapi.readthedocs.io/

${ }^{13}$ http://facom.ufms.br/ cafeo/vem2020-reputation/
} 
Perguntas e Respostas. Em relação às perguntas, foi obtido um total de 549 perguntas do SO, sendo que 453 estavam relacionadas aos code samples do Android, 54 relacionadas aos code samples do Spring Boot, apenas 6 dos code samples do AWS e 36 perguntas relacionadas com code samples do Azure. A partir desse conjunto de perguntas foram identificadas 639 respostas, sendo que 538 respostas estão relacionadas aos code samples do Android, 62 relacionadas aos code samples do Spring Boot, 5 de code samples do AWS e 34 ao Azure. Vale ressaltar que o SO possui o conceito de "resposta aceita". Respostas aceitas são respostas que foram escolhidas pelo usuário que criou a pergunta como sendo a resposta correta. Com isso, também foram analisadas as respostas aceitas (145).

Reputação da comunidade. A reputação é uma métrica do SO que indica o quanto a comunidade acredita em um desenvolvedor ${ }^{14}$. Com o objetivo de medir o quanto um desenvolvedor tem de experiência, foram extraídas as reputações de todos os desenvolvedores que possuíam perguntas e respostas relacionadas aos code samples como descrito anteriormente. Além disso, para obter uma base de comparação com os outros desenvolvedores, foi extraída a reputação de todos os desenvolvedores da plataforma. A obtenção desses dados foi feita por meio da plataforma Stack Exchange ${ }^{15}$ que disponibiliza uma base de dados referentes à reputação de todos os 12.485.155 desenvolvedores do SO. Os dados obtidos de reputação do desenvolvedores comum foram: Mínimo (1), Máximo (1185733), Média (119), Mediana (1) e Moda (1).

\section{Resultados e Discussão}

Nesta seção, são apresentados os resultados e suas implicações obtidos ao longo da pesquisa. Na Seção 4.1 são apresentados os resultados e as implicações relacionados à $Q 1$ e a Seção 4.2 mostra os resultados e as implicações relacionadas à $Q 2$.

\subsection{Q1 - Qual a reputação dos desenvolvedores que perguntam sobre code samples no Stack Overflow?}

Do novato ao mais experiente. De maneira geral, nota-se um amplo intervalo de reputação dos desenvolvedores com a análise dos dados obtidos. Foram encontrados desde desenvolvedores novatos com reputação próxima de zero até os mais experientes com reputação maior que 2.000. Isso indica que, diferentemente do senso comum, code samples, apesar de sua simplicidade, podem ter como público-alvo inclusive desenvolvedores mais experientes.

Para cada público-alvo, uma estratégia. A Figura 3 mostra um gráfico de violino com box plot representando a reputação dos desenvolvedores que perguntam sobre os code samples no SO. Os desenvolvedores Android na mediana possuem reputação de 323. Esses desenvolvedores possuem maior reputação que a média da comunidade (119). O mesmo comportamento acontece ao analisar os code samples do Spring Boot $(430,5)$ e AWS $(388,5)$. Esses números indicam que os desenvolvedores que utilizam os code samples são mais experientes que a média da comunidade do SO. Com isso, acredita-se que esses code samples podem gerar muitas perguntas de desenvolvedores da comunidade ou até desencorajar o uso do produto. Diferentemente dos desenvolvedores que utilizam

\footnotetext{
${ }^{14}$ https://stackoverflow.com/help/whats-reputation

15 https://archive.org/download/stackexchange/stackoverflow.com-Users. $7 z$
} 
code samples do Android, AWS e Spring Boot, os desenvolvedores que utilizam os code samples da Azure possuem 57 de reputação na mediana, ou seja, mais baixa que a média da comunidade (119). Isso pode indicar que desenvolvedores que buscam os code samples da organização ou especificamente da Azure não são tão experientes. Dessa forma, a organização pode, por exemplo, focar na criação de outros materiais educativos antes de direcioná-los a um code sample.

Simplicidade não é tudo. Ainda na Figura 3, é observado que os desenvolvedores que utilizam code samples do Spring Boot são os que possuem maior reputação na mediana com 430,5. Ou seja, os desenvolvedores com maior reputação entre os code samples estudados. No entanto, nota-se que seus code samples possuem o menor número de linhas de código por arquivo Java com apenas 20,71 (Figura 1b), e também são os mais simples em termos de complexidade ciclomática ${ }^{16}$ (Figura 1c). Nesse caso, mesmo com os code samples aparentemente seguindo diretrizes e boas práticas, eles não foram suficientemente explicativos para o público-alvo com reputação acima da média. Nesse caso, nota-se que outros fatores, além das propriedades de código e projeto, devem ser consideradas na criação e manutenção dos code samples. Para entender esses fatores, seria importante explorar a razão das dúvidas. Por exemplo, as dúvidas podem ser relativas ao ambiente de trabalho, clonagem do repositório ou complexidade de uso do produto.

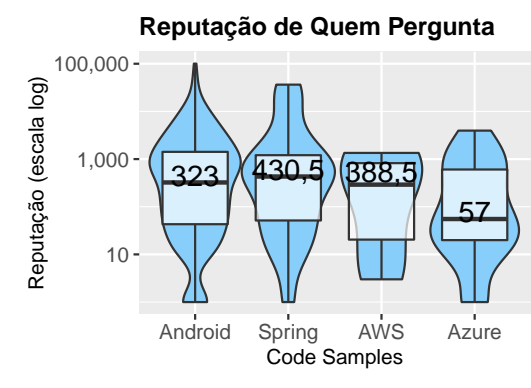

Figura 3. Reputação dos usuários que perguntam

\subsection{Q2 - Qual a reputação dos desenvolvedores que respondem as perguntas sobre os code samples no Stack Overflow?}

A comunidade importa. Um fato relevante é que, de forma geral, quem responde às perguntas sobre code samples têm maior reputação do que quem pergunta. No Android a reputação de quem pergunta é 323 e de quem responde é 885 . No Spring Boot tem-se reputação de 430,5 para quem faz uma pergunta sobre code sample e 1.387 para quem responde à essas perguntas. Isso se estende para o AWS no qual os quem pergunta têm reputação de 388,5, enquanto quem responde tem 646. Por fim, o Azure tem apenas 57 de reputação para quem pergunta contra 1.391 para quem responde. Isso pode reforçar a hipótese de que existem desenvolvedores mais experientes para responder as perguntas sobre code samples. Ou seja, o público-alvo do code sample não pode se limitar a um intervalo restrito em termos de experiência dos desenvolvedores, mas sim em um intervalo um pouco mais amplo. Dessa forma, a probabilidade de que dúvidas sejam sanadas pelos próprios participantes do ECOS é maior. Com o intervalo de reputação de quem pergunta e responde, a organização tem uma definição do público-alvo que deve focar quando for criar ou manter um code samples.

\footnotetext{
${ }^{16}$ Complexidade ciclomática é o número de caminhos de controle, aqui sendo extraída por método.
} 

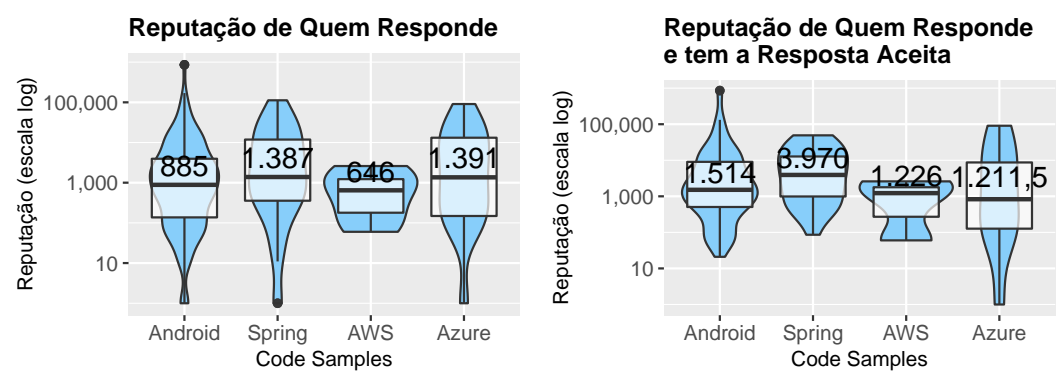

Figura 4. Reputação dos usuários que possuem resposta aceita

Seja pró-ativa. A Figura 4 mostra a reputação de quem tem resposta aceita pelo desenvolvedor que perguntou. As respostas aceitas são aquelas escolhidas pelo autor da pergunta como a que melhor atendeu às suas necessidades e geralmente são as que mais auxiliam outros desenvolvedores com a mesma dúvida. Observa-se que no Android a reputação de quem tem a resposta aceita é de 1.514, ou seja, maior que a reputação de quem apenas responde que é de 885. O mesmo acontece nos code samples do Spring Boot, tem-se 1.387 de reputação para quem responde contra 3.970 para os desenvolvedores que têm a resposta aceita. O AWS possui o mesmo comportamento aproximado: quem tem a resposta aceita tem mais reputação do que quem apenas responde, sendo 1.226 e 646, respectivamente. Esse fato é um indício de que a organização deve ser pró-ativa e engajar desenvolvedores mais experientes, evangelistas ou até desenvolvedores da organização que possam sanar dúvidas de outros desenvolvedores em sites de Q\&A. A única exceção feita nesse caso é ao Azure. Quem apenas responde tem a reputação maior do que quem têm a resposta aceita, 1.391 para quem responde e 1.211,5 para quem tem a resposta aceita.

\section{Trabalhos Relacionados}

Code samples. A pesquisa de Menezes e colegas [Menezes et al. 2019] realiza um estudo exploratório, buscando esclarecer aspectos encontradas em code samples, bem como definindo um conjunto de lições apreendidas. No trabalho de Zhang e colegas [Zhang et al. 2019], é proposta uma abordagem para o enriquecimento da documentação de API, mapeando code samples em cenários de uso correspondentes. Apesar dos trabalhos citados focarem em code samples, eles não abordam aspectos dos desenvolvedores que buscam ou usam esses projetos. Nesse trabalho, os desenvolvedores que utilizam code samples são explorados, evidenciando o grau de expertise deles no SO.

Reputação em sites de Q\&A. Ao contrário deste trabalho, que faz análise do públicoalvo de code samples pela reputação, o trabalho de Movshovitz-Attias e colegas [Movshovitz-Attias et al. 2013], foca no padrão de comportamento de usuários de alta e baixa reputação, concluindo que, embora a maioria das perguntas realizadas partam de usuários com baixa reputação, em média, um usuário de alta reputação realiza mais perguntas que um usuário de baixa reputação. No estudo realizado por Bosu e colegas [Bosu et al. 2013] é apresentada uma avaliação empírica sobre a reputação do usuário do SO, disponibilizando orientações a novos usuários para obterem altos índices de reputação de forma ágil. Diferentemente, neste trabalho, realiza-se a observação da reputação dos desenvolvedores que utilizam diversos code samples no SO, expondo possíveis contribuições às organizações no aprimoramento de seus repositórios e tornando-os mais adaptáveis às características dos desenvolvedores que os utilizam. 


\section{Conclusão e Trabalhos Futuros}

Baseado nos resultados obtidos com a condução do estudo exploratório, destaca-se os seguintes resultados: (1) Ao contrário do que se imaginava, os desenvolvedores que perguntam ou respondem sobre code samples são mais experientes que a comunidade em geral; (2) Code samples provavelmente são usados por uma grande variedade de desenvolvedores que vão desde novatos até os mais experientes; (3) Organizações devem buscar características em seus code samples compatíveis com a experiência da média da comunidade para evitar alto número de perguntas; (4) Organizações devem analisar os code samples individualmente em algumas situações para identificar público-alvo específico (por exemplo, menos ou mais experientes que a média), e assim adaptá-los a esses desenvolvedores; (5) Organizações devem engajar desenvolvedores experientes em seus code samples para que possam sanar as dúvidas de outros desenvolvedores em sites de Q\&A.

Como trabalhos futuros, planeja-se melhorar esta pesquisa ao avaliar dados qualitativos quanto aos desenvolvedores que utilizam code samples, entendendo suas maiores limitações e benefícios. Utilizar outros indicadores de experiência de usuários e, por fim, relacionar perguntas e respostas às características de código e projeto dos code samples.

Agradecimentos. Os autores agradecem a FACOM/UFMS, CAPES (código de financiamento 001) e CNPq (processos 438017/2018-8 e 133898/2020-2) pelo apoio financeiro.

\section{Referências}

Amreen, S., Karnauch, A., and Mockus, A. (2019). Developer reputation estimator (dre). In 2019 34th IEEE/ACM International Conference ASE, pages 1082-1085. IEEE.

Bosch, J. (2009). From software product lines to software ecosystems. In SPLC, volume 9, pages 111-119.

Bosu, A., Corley, C. S., Heaton, D., Chatterji, D., Carver, J. C., and Kraft, N. A. (2013). Building reputation in stackoverflow: An empirical investigation. In 2013 10th Working Conference MSR, pages 89-92.

Fontão, A., Lima, F., Ábia, B., dos Santos, R. P., and Dias-Neto, A. C. (2017). Hearing the voice of developers in mobile software ecosystems. In Proceedings of the 31st Brazilian Symposium on Software Engineering, SBES'17, page 4-13, New York, NY, USA. Association for Computing Machinery.

Menezes, G., Cafeo, B., and Hora, A. (2019). Framework code samples: How are they maintained and used by developers? In 2019 ACM/IEEE International Symposium ESEM, pages 1-11. IEEE.

Morrison, P. and Murphy-Hill, E. (2013). Is programming knowledge related to age? In Companion to the Working Conference MSR, pages 1-4. Citeseer.

Movshovitz-Attias, D., Movshovitz-Attias, Y., Steenkiste, P., and Faloutsos, C. (2013). Analysis of the reputation system and user contributions on a question answering website: Stackoverflow. In 2013 IEEE/ACM International Conference on ASONAM 2013, pages 886-893. IEEE.

Zhang, J., Jiang, H., Ren, Z., Zhang, T., and Huang, Z. (2019). Enriching api documentation with code samples and usage scenarios from crowd knowledge. IEEE Transactions on Software Engineering. 